\title{
Molecular Gas Excitation and Chemistry in VV 114 and NGC 1614 with ALMA
}

Toshiki Saito ${ }^{1,2}$, Daisuke Iono ${ }^{2,3}$, Junko Ueda ${ }^{2,4}$, Min S. Yun $^{5}$, Kouichiro Nakanishi ${ }^{2,3}$, Hajime Sugai ${ }^{6}$, Masatoshi Imanishi ${ }^{2,3,7}$, Yoshiaki Hagiwara ${ }^{8}$, Hiroyuki Kaneko ${ }^{2,9}$, Kentaro Motohara ${ }^{10}$, Daniel Espada $^{11}$, Takuji Yamashita ${ }^{12}$, Minju Lee ${ }^{1,2}$, Tomonari Michiyama ${ }^{2,3}$, and Ryohei Kawabe ${ }^{1,2,3}$

${ }^{1}$ Department of Astronomy, Graduate school of Science, The University of Tokyo, 7-3-1 Hongo, Bunkyo-ku, Tokyo 133-0033, Japan email: toshiki.saito@nao.ac.jp

${ }^{2}$ National Astronomical Observatory of Japan, 2-21-1 Osawa, Mitaka, Tokyo 181-8588, Japan

${ }^{3}$ Department of Astronomical Science, SOKENDAI (The Graduate University for Advanced Studies), 2-21-1 Osawa, Mitaka, Tokyo 181-8588, Japan

${ }^{4}$ Harvard-Smithsonian Center for Astrophysics, 60 Garden Street, Cambridge, MA 02138, USA

${ }^{5}$ Department of Astronomy, University of Massachusetts, Amherst, MA 01003, USA

${ }^{6}$ Kavli Institute for the Physics and Mathematics of the Universe (WPI),

The University of Tokyo, 5-1-5 Kashiwanoha, Kashiwa, Chiba 277-8583, Japan

${ }^{7}$ Subaru Telescope, 650 North A'ohoku Place, Hilo, HI 96720, USA

${ }^{8}$ Natural Science Laboratory, Toyo University, 5-28-20, Hakusan, Bunkyo-ku, Tokyo 112-8606, Japan

${ }^{9}$ Nobeyama Radio Observatory, NAOJ, 462-2 Minamimaki, Minamisaku, Nagano, 384-1305, Japan

${ }^{10}$ Institute of Astronomy, The University of Tokyo, 2-21-1 Osawa, Mitaka, Tokyo 181-0015, Japan

${ }^{11}$ Joint ALMA Observatory, Alonso de Córdova 3107, Vitacura, Casilla 19001, Santiago 19, Chile

${ }^{12}$ Institute of Space and Astronautical Science, Japan Aerospace Exploration Agency, 3-1-1 Yoshinodai, Sagamihara, Kanagawa 229-8510, Japan

\begin{abstract}
We present high resolution molecular line observations of dusty AGN and starburst in nearby luminous infrared galaxies (LIRGs), VV 114 (band 3/4/7) and NGC 1614 (band 3/6/7/9), with ALMA. Multi-frequency imaging from $4.8 \mathrm{GHz}$ to $691 \mathrm{GHz}$ of NGC 1614 allows us to study spatial properties of the radio-to-FIR continuum and multiple CO transitions, and we find the $\mathrm{CO}$ excitation up to $J_{\text {upp }}=6$ can be explained by a single ISM model powered by nuclear starbursts. Our processing line imaging survey for VV 114 detected at least 30 molecular lines which show different chemical composition from region to region. Multi-molecule imaging helps us to diagnose the chemical differences of dusty ISM, while multi-transition imaging allows us to investigate gas physical conditions affected by nuclear activities directly.
\end{abstract}

Keywords. Galaxies: interaction - Galaxies: starburst - ISM: molecules

\section{NGC 1614: CO SLED and Radio-to-FIR SED}

NGC 1614 is one of nearby $\left(D_{\mathrm{L}}=68.6 \mathrm{Mpc}\right)$ LIRGs $\left(L_{\mathrm{IR}}=10^{11.65} L_{\odot}\right.$; Armus et al. 2009), which is thought to be a minor merger remnant with an intense starburst ring (no strong AGNs; Herrero-Illana et al. 2014). The CO (6-5) has a completely different morphology compared with the low- $J$ CO (Sliwa et al. 2014; Xu et al. 2015). We modeled the CO Spectral Line Energy Distribution (SLED) by RADEX (van der Tak et al. 2007). The observed CO SLED up to $J_{\text {upp }}$ $=6$ can be explained by single-phase ISM model. A modeling with PDR Toolbox (Kaufman et al. 2006) revealed that the CO excitations can be explained by intensities of the FUV radiation field 
without any AGN contributions. This is consistent with the radio-to-FIR SED of NGC 1614 which mainly come from star-forming activities. The CO SLED of NGC 1614 is powered by starburst activities. See Saito et al. (2015b) submitted and Saito et al. (2015c) in preparation in details.

\section{VV 114: 84 - $111 \mathrm{GHz}$ and 127 - $154 \mathrm{GHz}$ Molecular Line Survey}

VV 114 is a mid-stage merger $\left(L_{\mathrm{IR}}=10^{11.71} L_{\odot}\right)$. The eastern galaxy has a strong hard X-ray nucleus (putative AGN) surrounded by soft X-ray/MIR/Paschen $\alpha$ emission (Le Floc'h et al. 2002; Grimes et al. 2006). The western galaxy is an UV-bright galaxy because of less amounts of dust. Cycle 0 ALMA observations revealed that the eastern nucleus shows several dense gas clumps with various $\mathrm{HCN}(4-3) / \mathrm{HCO}^{+}(4-3)$ ratios which are used for the extinction-free AGN diagnostic ( $>1$ indicates a dusty AGN signature). The $\mathrm{CH}_{3} \mathrm{OH}\left(2_{k}-1_{k}\right)$ emission is only detected at the "overlap region" where is located between the progenitor's disks. Our processing cycle 2 line survey preliminary detected rare species such as $\mathrm{CCH}, \mathrm{HNCO}, \mathrm{HNC}, \mathrm{CH}_{3} \mathrm{OH}, \mathrm{HC}_{3} \mathrm{~N}$, $\mathrm{H}_{2} \mathrm{CS}, \mathrm{CH}_{3} \mathrm{CCH}, \mathrm{H}_{2} \mathrm{CO}$, and $c-\mathrm{C}_{3} \mathrm{H}_{2}$. We find that these detected molecules are different from region to region (e.g., multiple transitions of $\mathrm{CH}_{3} \mathrm{OH}$ and $\mathrm{HNCO}$, which are commonly used for extragalactic shock tracers, are only detected at the overlap region), suggesting underlying chemistry is also different. Gas chemistry can distinguish the AGN at the eastern nucleus, starburst activities, and the overlap region (putative shocked region due to the collision of gasrich galaxies) between the galaxy disks. See Iono et al. (2013), Saito et al. (2015a), and Saito et al. (2016) in preparation in details.

\section{References}

Armus, L., Mazzarella, J. M., Evans, A. S., Surace, J. A., Sanders, D. B., Iwasawa, K., Frayer, D. T., Howell, J. H., Chan, B., Petric, A., Vavilkin, T., Kim, D. C., Haan, S., Inami, H., Murphy, E. J., Appleton, P. N., Barnes, J. E., Bothun, G., Bridge, C. R., Charmandaris, V., Jensen, J. B., Kewley, L. J., Lord, S., Madore, B. F., Marshall, J. A., Melbourne, J. E., Rich, J., Satyapal, S., Schulz, B., Spoon, H. W. W., Sturm, E., U, V., Veilleux, S., Xu, K. 2009, PASP, 121, 559

Grimes, J. P., Heckman, T., Hoopes, C., Strickland, D., Aloisi, A., Meurer, G., Ptak, A. 2006, ApJ, 648, 310

Herrero-Illana, R., Pérez-Torres, M. Á., Alonso-Herrero, A., Alberdi, A., Colina, L., Efstathiou, A., Hernández-García, L., Miralles-Caballero, D., Väisänen, P., Packham, C. C., Rajpaul, V., Zijlstra, A. A. 2014, ApJ, 786, 156

Iono, D., Saito, T., Yun, M. S., Kawabe, R., Espada, D., Hagiwara, Y., Imanishi, M., Izumi, T., Kohno, K., Motohara, K., Nakanishi, K., Sugai, H., Tateuchi, K., Tamura, Y., Ueda, J., Yoshii, Y. 2013, PASJ (Letters), 65, L7

Kaufman, M. J., Wolfire, M. G., \& Hollenbach, D. J. 2006, ApJ, 644, 283

Le Floc'h, E., Charmandaris, V., Laurent, O., Mirabel, I. F., Gallais, P., Sauvage, M., Vigroux, L., Cesarsky, C. 2002, A\& $A, 391,417$

Saito, T., Iono, D., Yun, M. S., Ueda, J., Nakanishi, K., Sugai, H., Espada, D., Imanishi, M., Motohara, K., Hagiwara, Y., Tateuchi, K., Lee, M., Kawabe, R. 2015, ApJ, 803, 60

Sliwa, K., Wilson, C. D., Iono, D., Peck, A., Matsushita, S. 2014, ApJ (Letters), 796, L15

van der Tak, F. F. S., Black, J. H., Schöier, F. L., Jansen, D. J., van Dishoeck, E. F. 2007, A\&A, 468,627

Xu, C. K., Cao, C., Lu, N., Gao, Y., Diaz-Santos, T., Herrero-Illana, R., Meijerink, R., Privon, G., Zhao, Y.-H., Evans, A. S., König, S., Mazzarella, J. M., Aalto, S., Appleton, P., Armus, L., Charmandaris, V., Chu, J., Haan, S., Inami, H., Murphy, E. J., Sanders, D. B., Schulz, B., van der Werf, P. 2015, ApJ, 799, 11 